\title{
Análise dos encaminhamentos de pacientes cardiopatas adultos segundo o protocolo do Ministério da Saúde
}

\author{
Derivation analysis of adult patients with heart disease according to the Ministry of Health \\ protocol
}

\section{Análisis de derivaciones de pacientes adultos con cardiopatías según protocolo del Ministerio de Sanidad}

Bruno Lima Cintra1*, Michela Macedo Lima Costa ${ }^{1}$, Camila Vilasboas Oliveira Cardoso Leite ${ }^{1}$, João Victor Santana Araújo ${ }^{1}$, Enyo Arruda Santos ${ }^{1}$, Andressa Lima Porto ${ }^{1}$, Luísa Nery Matos ${ }^{1}$, Luana Maria Liborio da Mota ${ }^{1}$, Shesllen Mikaelly Cruz Corrêa ${ }^{1}$, Ricely Almeida Rezende'.

\section{RESUMO}

Objetivo: Analisar se há obediência aos critérios expostos no protocolo ao solicitar uma consulta com médico especialista em cardiologia. Métodos: Trata-se de uma análise documental, quantitativa e retrógrada das fichas de solicitação de encaminhamento e prontuários dos pacientes cardiopatas adultos que foram referenciados de uma Unidade de Saúde da Família (USF) para consulta com cardiologista no município de Vitória da Conquista - Bahia. Com base nos parâmetros do protocolo de encaminhamento em cardiologia (PEABEC) do Ministério da Saúde. Os dados colhidos foram digitalizados para planilha do software Microsoft Office Excel. O estudo foi aprovado por Comitê de Ética em Pesquisa. Resultados: A média de tempo de espera na fila foi de 498,3 dias para obtenção de nova consulta com cardiologista. A USF avaliada recebe em média três vagas mensais para esta finalidade. Dezesseis pacientes foram selecionados para avaliação documental com média de idade de 60,56 anos de modo que $50 \%$ era do sexo feminino e $43,75 \%$ masculino. Conclusão: Evidenciou-se uma lacuna nos registros de informações nos documentos analisados e no impacto negativo direto na priorização do acesso e na formação de longas filas.

Palavras-chave: Acesso aos serviços de saúde, Regulação e fiscalização em saúde, Atenção primária à saúde, Cardiologia.

\begin{abstract}
Objective: To analyze if there is compliance with the criteria set out in the protocol when requesting an appointment with a cardiology specialist. Methods: This is a documentary, quantitative and retrograde analysis of referral request forms and medical records of adult heart disease patients who were referred from a Family Health Unit (USF) for consultation with a cardiologist in the city of Vitória da Conquista - Bahia. Based on the parameters of the Cardiology Referral Protocol (PEABEC) of the Ministry of Health. The collected data were digitized into a spreadsheet in the Microsoft Office Excel software. The study was approved by the Research Ethics Committee. Results: The average waiting time in line was 498.3 days to obtain a new appointment with a cardiologist. The evaluated USF receives an average of three monthly places for this purpose. Sixteen patients were selected for documentary evaluation with a mean age of 60.56 years, $50 \%$ female and $43.75 \%$ male. Conclusion: There was evidence of a gap in the records of information in the documents analyzed and the direct negative impact on prioritizing access and the formation of long queues.
\end{abstract}

Key words: Access to health services, Regulation and supervision in health, Primary health care, Cardiology.

\section{RESUMEN}

Objetivo: Analizar si se cumplen los criterios establecidos en el protocolo a la hora de solicitar cita con un especialista en cardiología. Métodos: Se trata de un análisis documental, cuantitativo y retrógrado de formularios de solicitud de derivación y expedientes médicos de pacientes adultos con cardiopatías que fueron remitidos desde la Unidad de Salud de la Familia (USF) para consulta con un cardiólogo en la ciudad de Vitória da Conquista - Bahia. Basado en los parámetros del Protocolo de Derivación Cardiológica (PEABEC)

${ }^{1}$ Faculdade Santo Agostinho (FASA), Vitória da Conquista - BA. *E-mail: bwcintra@gmail.com 
del Ministerio de Salud. Los datos recopilados se digitalizaron en una hoja de cálculo en el software Microsoft Office Excel. El estudio fue aprobado por el Comité de Ética en Investigación. Resultados: El tiempo medio de espera en la cola fue de 498,3 días para obtener una nueva cita con un cardiólogo. La USF evaluada recibe una media de tres plazas mensuales para este fin. Se seleccionaron 16 pacientes para evaluación documental con una edad media de 60,56 años, $50 \%$ mujeres y 43,75\% hombres. Conclusión: Se evidenció una brecha en los registros de información en los documentos analizados y el impacto negativo directo en la priorización del acceso y la formación de largas colas.

Palabras clave: Acceso a los servicios de salud, Regulación y supervisión en salud, Atención primaria de salud, Cardiología.

\section{INTRODUÇÃO}

A organização do atual modelo de saúde evidencia os níveis de atenção quanto a uma série de fatores. Interpretar hoje a Atenção Primária à Saúde (APS) apenas como um serviço de boa resolutividade aos agravos frequentes da população é uma visão simplista deste setor (XIMENES NETO FRG, et al., 2019). No atual modelo de gestão do SUS, a atenção primária, deve ser interpretada como uma estratégia de organização do sistema de saúde, sendo essa uma forma de apropriar, recombinar e reordenar os recursos do sistema, afim de aplicar especificamente para a população adscrita da melhor maneira possível, uma atenção primária eficiente, resolutiva e integrada com níveis secundários e terciários de atenção (EVANGELISTA MJDO, et al., 2019).

Cerca de $85 \%$ das pessoas que procuram assistência à saúde têm em seu agravo, resolutividade na atenção primária, enquanto que $10 \%$ têm necessidade de encaminhamento para especialista focal e outros $5 \%$ necessidade de serviços de alta complexidade tecnológica. Dessa maneira, estes números traduzem a importância deste setor como centro resolutivo e ordenador das redes de atenção (MOROSINI MVGC, et al., 2018).

Ainda assim, quando processo de encaminhamento para serviços especializados (referenciamento) é realizado por unidades de saúde da APS sem a necessidade adequada, evidencia certa fragilidade dos mecanismos reguladores da rede, da gestão clínica, das condições de acesso ao serviço e recursos humanos. Além dos sistemas de informação e comunicação e apoio logístico, que são elementos fundamentais para promoção da integralidade da rede (SERRA CG e RODRIGUES PHDA, 2010).

Outra situação particular, no que tange, ao sistema de saúde, é a transição epidemiológica que vive o Brasil (BAHIA L, 2018). De modo que o perfil das doenças têm-se invertido quando comparados ao início do século $X X$, em que no padrão de adoecimento e mortalidade da população observa-se um aumento importante da prevalência das doenças crônicas não transmissíveis (DCNT) (MALTA DC, et al., 2017). Um fator interessante, é que as doenças do aparelho circulatório lideraram as causas de mortalidade no Brasil no ano de 2012. Nesse ínterim, nota-se um predomínio cada vez maior das DCNT na população brasileira com a evolução do tempo, o que marca o perfil dos atendimentos na APS (PEITER CC, et al., 2019).

Desta forma, o Ministério da Saúde (MS) a partir do ano de 2016 dispôs da criação de protocolos de encaminhamento, para que estes sirvam de base para as equipes de saúde que trabalham na APS, como suporte técnico de quando e como encaminhar o paciente ao serviço especializado e em que momento manejar esse paciente no âmbito do serviço primário de atenção (BASTOS LBR, et al., 2020). Nesta condição, um protocolo específico para o paciente cardiopata adulto foi elaborado visto que a demanda destes pacientes na APS é elevada, e consequentemente seus aspectos no sistema de referência e contrarreferência da rede devem ser bem criteriosos (BERNARDINO JUNIOR SV, et al., 2020).

Diante do exposto, nota-se que apesar do protocolo de encaminhamento estar em vigência desde 2016, existe a possibilidade de haver um referenciamento inadequado de pacientes cardiopatas para o serviço especializado. Com isso, esse processo pode ocasiona longas filas de espera e demanda reprimida nas unidades de saúde, evidenciando dois possíveis desfechos: ou a demanda é realmente alta ou a resolutividade da APS está deficitária (MELO EA, et al., 2021). Esse estudo visou analisar se há obediência aos critérios expostos no protocolo ao solicitar uma consulta com médico especialista em cardiologia. 


\section{MÉTODOS}

O presente estudo trata-se de uma análise documental, quantitativa e retrógrada das fichas de solicitação de encaminhamento e prontuários dos pacientes cardiopatas adultos que foram referenciados de uma Unidade de Saúde da Família (USF) situada na região urbana do município de Vitória da Conquista, no estado da Bahia, para consulta especializada com o médico cardiologista na rede de atenção municipal. A pesquisa foi realizada no mês de setembro de 2020 após aprovação do Comitê de Ética em Pesquisa da Fundação Pública de Saúde de Vitória da Conquista (CEP/FSVC) segundo o parecer de número 4.141647.

Os critérios de inclusão para análise desses documentos foram: os pacientes que tiveram solicitação para encaminhamento mas que não foram encaminhados, desde que solicitado em 2019; os pacientes deveriam ser adultos com idade superior à vinte e um anos na época da solicitação; ser portadores de pelo menos uma das afecções cardiovasculares abordadas pelo Protocolo De Encaminhamento Da Atenção Básica Para Especializada Em Cardiologia (PEABEC), que, a saber, são a Cardiopatia Isquêmica (Cl), a Insuficiência Cardíaca (IC), as arritmias, a Síncope Ou Perda Transitória Da Consciência (SPTC), a Hipertensão Arterial Sistêmica (HAS) e as Valvopatias (VPT). Os pacientes em que não foi possível identificar a patologia do encaminhamento também foram incluídos (BRASIL, 2016).

Dessa forma, excluiu-se da pesquisa os pacientes que possuíam o diagnóstico de outras patologias que não as supracitadas; os que não foram solicitados para encaminhamento no intervalo de tempo escolhido; os documentos ilegíveis; os pedidos de retorno; os pedidos sem data registrada e os pedidos repetidos. $O$ instrumento utilizado para colher os dados, foi o documento denominado "Ficha de Análise do Encaminhamento do Paciente para Cardiologia Clínica", construído pela equipe de pesquisa. Neste documento, constavam os dados considerados necessários para encaminhar um paciente ao cardiologista na cobertura do Sistema Único de Saúde (SUS) e analisava, de acordo com o PEABEC, as condições clínicas que foram documentadas pela equipe no momento da solicitação (BRASIL, 2016).

Assim, ao ter acesso aos documentos, cada tópico da Ficha de Análise do Encaminhamento do Paciente para Cardiologia Clínica, foi preenchido manualmente pelos pesquisadores. As informações colhidas foram: sexo, idade, patologia cardiovascular apresentada, motivo da solicitação, data que a solicitação foi realizada, se o encaminhamento foi ou não realizado, e em caso de resposta positiva, quando foi realizado. Além disso, a ficha também foi preenchida com os sinais e sintomas apresentados pelo paciente, medicamentos em uso e resultados de exames, bem como com outros itens que são particulares de cada cardiopatia.

Após preenchimento de todas as fichas, os dados colhidos foram digitalizados para planilha do software Microsoft Office Excel @ versão 2016 MSO, e posteriormente foram classificados de acordo aos critérios estabelecidos pelo PEABEC (BRASIL, 2016). Os pesquisadores, definiram então, de acordo com a quantificação dos itens que foram documentados, em conformidade com itens descritos no PEABEC como "conteúdo descritivo mínimo", quatro grupos de classificação (Tabela 1).

Tabela 1 - Grupos de classificação dos encaminhamentos ao cardiologista

\begin{tabular}{cl}
\hline Grupo de Classificação & \multicolumn{1}{c}{ Parecer } \\
\hline A & $\begin{array}{l}\text { A ficha de solicitação de consulta com cardiologista contém todos os itens } \\
\text { preenchidos de acordo com o protocolo. }\end{array}$ \\
\hline B & $\begin{array}{l}\text { Ficha de solicitação de consulta com cardiologista não tinha todos os critérios } \\
\text { necessários para realizar o encaminhamento de acordo com protocolo, no } \\
\text { entanto encontrou-se os demais itens descritos em prontuário. }\end{array}$ \\
\hline C & $\begin{array}{l}\text { Tanto a ficha de solicitação de consulta com cardiologista quanto o prontuário } \\
\text { do paciente não tinham todos os critérios descritos que justificassem o } \\
\text { encaminhamento de acordo com o protocolo. }\end{array}$ \\
\hline D & $\begin{array}{l}\text { Dados insuficientes ou ilegíveis para avaliação da ficha de solicitação de } \\
\text { consulta com cardiologista e/ou do prontuário. }\end{array}$ \\
\hline
\end{tabular}

Fonte: Cintra BL, et al., 2021. 


\section{RESULTADOS}

A pesquisa foi dividida em três fases, a primeira fase do trabalho contabilizou um total de 55 fichas de solicitação de encaminhamento para o médico cardiologista. Destas a maioria eram para novas consultas $(n=32)$ e os demais eram pedidos de retorno $(n=23)$. Das solicitações de nova consulta, prosseguiram para contabilização do tempo de espera na fila (TEF) 26 fichas, pois foram descartadas seis pelos seguintes motivos: conteúdo ilegível $(n=1)$; paciente com idade inferior a 21 anos $(n=1)$, paciente repetido $(n=1)-$ foi considerado o pedido mais antigo - e ficha sem data de solicitação $(n=3)$, dados ilustrados na (Tabela 2).

Das 26 fichas de solicitação que foram utilizadas para calcular o TEF, duas eram do ano de 2017, seis do ano de 2018, dezesseis do ano de 2019 e duas do ano de 2020. Dessa forma, a média de tempo de espera foi de 498,3 dias, de modo que o pedido mais antigo aguardando uma vaga de nova consulta está há 1.046 dias, e o que espera há menos tempo na fila, aguarda há 197 dias.

Tabela 2 - Quantidade de fichas de acordo com as categorias dos critérios de inclusão e exclusão

\begin{tabular}{lc}
\hline $\begin{array}{l}\text { Contabilização das fichas de solicitação de acordo com as diferentes } \\
\text { categorias }\end{array}$ & "n” \\
\hline Total de fichas de solicitação de consulta com o cardiologista retidas & 55 \\
\hline Total de fichas de solicitação de consulta de retorno & 23 \\
\hline Total de fichas de solicitação de nova consulta & 32 \\
\hline Total de fichas descartadas & 29 \\
\hline Descartadas pois o paciente apresentava idade inferior a 21 anos & 1 \\
\hline Descartadas por conta da ausência de data na ficha de solicitação & 3 \\
\hline Descartadas por haver mais de um pedido para o mesmo paciente (sendo & 1 \\
considerado apenas o pedido mais antigo) & 1 \\
\hline Descartadas por serem ilegíveis & 23 \\
\hline Descartadas por serem solicitação de consulta de retorno & 26 \\
\hline Fichas aprovadas para evolução na pesquisa por ano & 2 \\
\hline Fichas de novas consultas ano 2017 & 6 \\
\hline Fichas de novas consultas ano 2018 & 16 \\
\hline Fichas de novas consultas ano 2019 & 2 \\
\hline Fichas de novas consultas ano 2020 & \\
\hline
\end{tabular}

Fonte: Cintra BL, et al., 2021.

A segunda fase da pesquisa que envolveu a contabilização da oferta de vagas mensais de consulta com o cardiologista para a USF de escolha no ano de 2019 foi avaliada de acordo com a quantidade de pacientes descritos no caderno de registro de procedimentos especializados. Todavia, por conta da metodologia do registro da equipe foi um dado de difícil obtenção, de modo que só foi possível encontrar o número de vagas para nova consulta e retornos em quatro meses do ano de 2019. Obteve-se então uma média de três vagas mensais para novas consultas e 3,25 para consultas de retorno.

A terceira fase que se aplica à análise das fichas de solicitação e aos prontuários dos pacientes considerando a quantidade de itens descritos de acordo com o PEABEC, analisou ao todo dados dos dezesseis pacientes que estavam com a ficha retida no ano de 2019, mantendo os critérios de inclusão das fases anteriores (BOUSQUAT A, et al., 2017). O perfil dos pacientes analisados fora de $50 \%$ mulheres e $43,75 \%$ de homens, de modo que em $6,25 \%(n=1)$ não foi possível identificar o sexo do paciente. A média de idade destes foi de 60,56 anos.

O resultado da quantificação do preenchimento das fichas e do prontuário seguiu da seguinte forma: $56,25 \%$ dos documentos se classificaram no grupo $C(n=9), 31,25 \%$ se classificaram no grupo $D(n=5), 12,5 \%$ se classificaram no grupo $B(n=2)$ e nenhum dos documentos avaliados conseguiram classificação no grupo 
A ( $0 \%$ ). De modo que os pacientes que se classificaram como $D$, em $80 \%(n=4)$ não foi possível a obtenção do prontuário e $20 \%(n=1)$ os itens descritos tanto em ficha de solicitação quanto no prontuário eram insuficientes. Esses dados são abordados no (Gráfico 1).

Gráfico 1 - Percentual dos grupos de classificação dos encaminhamentos ao cardiologista.

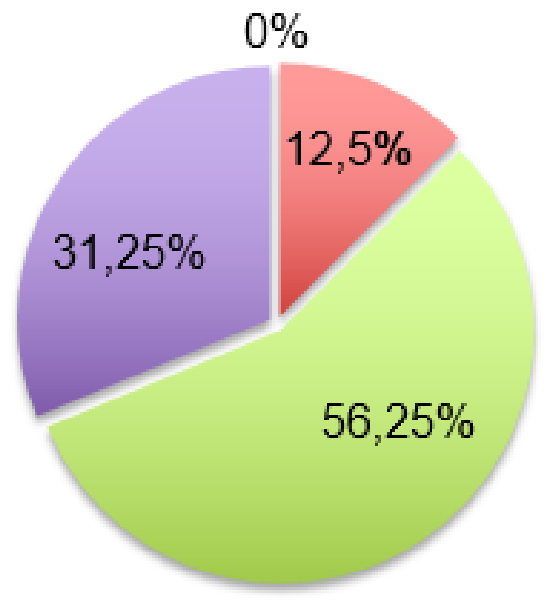

॥ Classificação A

• Classificação B

Classificação C

• Classificação D

Fonte: Cintra BL, et al., 2021.

Além disso, os pacientes foram classificados nos blocos das cardiopatias de acordo com o que estava descrito na ficha de solicitação e no prontuário, de modo que $56,25 \% \quad(n=9)$ foram associados à encaminhamento por Hipertensão Arterial Sistêmica, em 18,75\% ( $n=3)$ dos documentos não foi possível classificar o paciente, $6,25 \%$ foram associados à encaminhamento por Arritmias, 6,25\% ( $n=1)$ foram associados à encaminhamento por Cardiopatia Isquêmica, $6,25 \%(n=1)$ foram associados à encaminhamento por Insuficiência Cardíaca e 6,25\% (n=1) foram associados à encaminhamento por Valvopatias.

Os motivos das solicitações de consulta que estavam escritos nas fichas de solicitação estão identificados na (Quadro 1). De modo que nenhum motivo de solicitação descrito na ficha estava correspondente com a indicação de consulta do PEABEC (BOUSQUAT A, et al., 2017).

Quadro 1 - Motivos escritos nas fichas de solicitação avaliadas na terceira fase (ipsis litteris).

\begin{tabular}{|l|}
\hline Motivo da solicitação de consulta com cardiologista - fichas da 3a fase \\
\hline Hipertensão Arterial \\
\hline Dor Precordial Atípica \\
\hline HAS + HVE \\
\hline HAS / Bradicardia / Sobrecarga Atrial Esquerda \\
\hline HAS + Obesidade \\
\hline Cardiopatia - exames \\
\hline Hipertensão Arterial / Sobrecarga Ventricular Esquerda \\
\hline DPOC + IC? \\
\hline HAS não controlada \\
\hline HAS severa / difícil controle \\
\hline Sobrecarga Ventricular Esquerda \\
\hline IC / DM \\
\hline
\end{tabular}

Legenda: HAS- Hipertensão Arterial Sistêmica, HVE- Hipertrofia Ventricular Esquerda, DPOC- Doença Pulmonar Obstrutiva Crônica, IC- Insuficiência Cardiaca, DM- Diabetes Mellitus. Fonte: Cintra BL, et al., 2021. 
Desta maneira, em $50 \%(n=8)$ dos pacientes que tiveram os documentos avaliados não foi possível identificar um motivo de acordo com o protocolo, neste grupo estão inclusos os quatro pacientes em que não foi possível acessar o prontuário. Em $18,75 \%(n=3)$ ao avaliar o prontuário os pacientes se enquadraram no motivo do protocolo "Hipertensão mal controlada (fora do alvo terapêutico) com no mínimo três medicações anti-hipertensivas em dose plena, após avaliar a adesão". Em $6,25 \%$ dos pacientes $(n=1)$ se enquadraram no motivo do PEABEC "Cardiopatia isquêmica estabelecida com necessidade de estratificação de risco do paciente após evento agudo (não realizado no serviço de urgência/emergência) ". E 6,25\% ( $n=1$ ) ao acessar o prontuário do paciente identificou-se que foi solicitado encaminhamento de acordo com o motivo do protocolo "Bloqueio bifascicular" (bloqueio completo de ramo esquerdo)".

A última análise levou em conta o que o protocolo refere como conteúdo descritivo mínimo para realização do encaminhamento. Ao separar os pacientes classificados como categoria D, ou seja, com dados insuficientes para a análise, observou-se que era necessário encontrar na ficha de solicitação e no prontuário de todos os pacientes 71 itens para realização do encaminhamento. Desses itens foram encontrados apenas $38(53,52 \%)$, de modo que apenas dois $(5,26 \%)$ desses itens foram descritos nas fichas de solicitação e 36 (94,73\%) foram identificados no prontuário.

É importante relatar que, dos 71 itens que analisados, 27 (38,02\%) eram itens que não foram considerados para classificação final nas categorias $A, B, C$ e D (itens relativos), os quais representaram oito dos 38 itens preenchidos nos documentos. Assim, dos 44 itens descritivos que foram considerados indispensáveis em um encaminhamento para uma consulta especializada com o médico cardiologista, apenas $30(68,18 \%)$ foram descritos, sendo que $29(96,66 \%)$ desses foram descritos apenas em prontuário, o que justifica o grande número de documentos categorizados como grupo de classificação $\mathrm{C}$.

\section{DISCUSSÃO}

Pode-se entender que é de conhecimento do próprio PEABEC que por conta do seu papel estratégico na RAS, é necessário que a atenção básica tenha uma alta resolutividade e isso depende de alguns fatores, dentre eles estão inclusos a capacidade clínica e de cuidado das equipes, as tecnologias duras disponíveis e a articulação com outros serviços (FRANCO TB e HUBNER LC, 2020). É importante salientar que o serviço especializado representa um componente deficitário das RAS, principalmente por ser de difícil acesso, o que ocasiona longas filas de espera em concomitância com poucas vagas disponíveis (SCHIMITH MD, et al., 2019). Esse aspecto foi evidenciado nos resultados da pesquisa, de modo que, os pacientes analisados estão esperando em média 498,3 dias por uma vaga de nova consulta com o médico cardiologista.

No município de Vitória da Conquista - BA, para realizar a regulação do acesso as vagas de exames, consultas e procedimentos especializados foi implementada no ano de 1999 a Central de Regulação de Procedimentos e Exames Especializados (CRPEE), que é o órgão responsável pela disponibilização dos serviços de média complexidade para população do município e zona rural, além de 73 outros municípios da macrorregião sudoeste da Bahia que pactuaram os procedimentos pelo Programação Pactuada Integrada (PPI) (SAMPAIO MVDA, 2016).

A CRPEE envia uma oferta mensal das vagas para as consultas com especialista para todas unidades de saúde da rede de atenção municipal, e as equipes de saúde das unidades por sua vez são responsáveis pela priorização e escolha de qual paciente será encaminhado para vaga ofertada (SANTOS L, 2017). Diante disso, a pesquisa encontrou uma média mensal de oferta para consulta com o médico cardiologista de 3 vagas para pacientes que necessitam de nova consulta e 3,25 para os pacientes que necessitam de retorno. É importante ressaltar que através do instrumento avaliado, só se pode encontrar esses números em quatro meses do ano de 2019, sendo assim calculada a média.

Pode-se observar que para o volume de pacientes encontrados aguardando vaga de nova consulta $(n=24)$, com o regime de oferta atual, ainda assim sobram em média oito pacientes por ano sem conseguir a vaga, visto que foram encontrados solicitações dos anos de 2017, 2018 e 2019, ou seja, não existe uma cobertura capaz de suprir a demanda da unidade de maneira adequada. Acentua-se que esse dado representa apenas 
uma insuficiência anual de vagas para nova consulta com cardiologista referente à uma unidade de saúde da família da rede de atenção municipal, esse número quando avaliado em uma ótica da oferta da atenção especializada pode representar um número mais expressivo.

Com base na Portaria oㅜ 1.559 de 1ํ de agosto de 2008 que institui a Política Nacional de Regulação do Sistema Único de Saúde - SUS, fazem parte da atribuição da regulação do acesso elaborar, disseminar e implantar protocolos de regulação bem como diagnosticar, adequar e orientar os fluxos de assistência. Dessa maneira o PEABEC foi elaborado com o objetivo de responder à duas principais questões para o médico responsável pela regulação e para equipes da atenção básica: 1 - O paciente tem indicação clínica para ser encaminhado? 2 - Quais os pacientes com indicação clínica que devem ser priorizados ao acesso? (BRASIL, 2016). É importante salientar que nenhum dos dezesseis encaminhamentos analisados que estavam aguardando vaga de nova consulta com o cardiologista seguiam de maneira adequada as indicações previstas no protocolo (classificação A). De modo que quatorze fichas de solicitação, documento indispensável ao encaminhamento de acordo com a Prefeitura Municipal de Vitória da Conquista (PMVC) (2020), continham apenas os motivos relatado no Quadro 1 que, em sua totalidade, quando comparados às indicações de consulta especializada do PEABEC, possuem motivos inespecíficos e de difícil estabelecimento de critério de priorização.

Dessa maneira, mesmo após a análise do prontuário, em $87,5 \%(n=14)$ dos documentos analisados o conteúdo obrigatório de descrição do PEABEC não foi alcançado ou mesmo não foi possível identificar nenhum dos dados (classificação $C$ e D), de maneira que é importante observar que em $25 \%$ dos pacientes avaliados o prontuário não foi encontrado. Ou seja, dos dezesseis pacientes avaliados, em apenas dois (12,5\%), encontrou-se os itens descritivos mínimos considerados obrigatórios no prontuário (classificação B). Além disso, grande quantidade de documentos classificados como categoria "C" é justamente que dos 44 itens considerados obrigatórios, apenas $30(68,18 \%)$ foram preenchidos sendo que desses, 96,6\% $(n=29)$ estavam registrados no prontuário.

É notória a repercussão negativa desses dados no que tange a priorização dos pacientes que terão acesso a consulta, de modo que estão há muito tempo na fila e não é possível identificar se há realmente necessidade de encaminhamento ou se a resolutividade das condutas da equipe da unidade avaliada se mostra abaixo do indicado. Ao não especificar as condições do encaminhamento na ficha não é possível evidenciar o real motivo da solicitação. Ou seja, esse déficit de esclarecimento do motivo pode ser um fator impactante na demora de acesso e priorização dos pacientes que podem vir a ter condições clínicas potencialmente complicáveis.

Dentre as dificuldades elencadas na organização das práticas de saúde estão: a falta de resolutividade da atenção básica, a demanda reprimida, a indefinição de fluxos de referência e contrarreferência e a carência de qualificação e capacitação de profissionais (SERRA CG e RODRIGUES PHDA, 2010). Pode-se observar a demanda reprimida com longas filas de espera, para finalidade dos pacientes avaliados (consulta com cardiologista) (DALLA LIBERA TC, et al., 2020). Outro aspecto evidenciado foi a carência de qualificação e capacitação de profissionais visto que se há uma inadequação evidente do preenchimento dos documentos necessários para referenciar o paciente ao serviço especializado e isso está causando dificuldade de acesso aos serviços, possivelmente a equipe não está familiarizada com as boas práticas do encaminhamento (OLIVEIRA CCRB, et al., 2021).

\section{CONCLUSÃO}

Diante dos resultados da pesquisa, pôde-se observar em totalidade o descumprimento dos critérios estabelecidos pelo protocolo de encaminhamento da atenção básica para a atenção especializada em cardiologia. De modo que isso ilustra uma lacuna de informações na descrição dos documentos dos pacientes e não estabelece por meio da avaliação dos registros realizados pela equipe à sua real condição clínica. Outro ponto foram os motivos descritos para solicitação da consulta com o especialista, que em sua totalidade foram inespecíficos e, em associação com o registro insatisfatório de dados clínicos considerados obrigatórios, impactam diretamente na priorização do acesso e na formação de longas filas. 


\section{REFERÊNCIAS}

1. BASTOS LBR, et al. Práticas e desafios da regulação do Sistema Único de Saúde. Revista de Saúde Pública, 2020; 54: 25.

2. BAHIA L. Trinta anos de Sistema Único de Saúde (SUS): uma transição necessária, mas insuficiente. Cadernos de Saúde Pública, 2018; 34: e00067218.

3. BRASIL. Protocolo de encaminhamento da atenção básica para a atenção especializada. 2016. Disponível em: http://bvsms.saude.gov.br/bvs/publicacoes/protocolos_atencao_basica_especializada_cardiologia_v_Il.pdf. Acessado em: 20 de novembro de 2020

4. BERNARDINO JUNIOR SV, et al. Processos de encaminhamento a serviços especializados em cardiologia e endocrinologia pela Atenção Primária à Saúde. Saúde em Debate, 2020; 44: 694-707.

5. BOUSQUAT $A$, et al. Atenção primária à saúde e coordenação do cuidado nas regiões de saúde: perspectiva de gestores e usuários. Ciência \& Saúde Coletiva, 2017; 22: 1141-1154.

6. DALLA LIBERA TC, et al. Análise comparativa entre estratégia de saúde da família e unidade básica de saúde na referência ao nível assistencial secundário em cardiologia. Manuscripta Médica, 2020; 3: 60-68.

7. EVANGELISTA MJDO, et al. O Planejamento e a construção das Redes de Atenção à Saúde no DF, Brasil. Ciência \& Saúde Coletiva, 2019; 24: 2115-2124.

8. FRANCO TB, HUBNER LC. A Atenção Básica e os cuidados intermediários: um debate necessário. Saúde em Debate, 2020; 44: 516-526.

9. MOROSINI MVGC, et al. Política Nacional de Atenção Básica 2017: retrocessos e riscos para o Sistema Único de Saúde. Saúde em Debate, 2018; 42: 11-24.

10. MELO EA, et al. A regulação do acesso à atenção especializada e a Atenção Primária à Saúde nas políticas nacionais do SUS. Physis: Revista de Saúde Coletiva, 2021; 31: e310109.

11. MALTA DC, et al. Doenças crônicas não transmissíveis e a utilização de serviços de saúde: análise da Pesquisa Nacional de Saúde no Brasil. Revista de Saúde Pública, 2017; 51:4.

12. OLIVEIRA CCRB, et al. Referência e contrarreferência para a integralidade do cuidado na Rede de Atenção à Saúde. Physis: Revista de Saúde Coletiva, 2021; 31: e310105.

13. PEITER CC, et al. Redes de atenção à saúde: tendências da produção de conhecimento no Brasil. Escola Anna Nery, 2019; 23:1.

14. PREFEITURA MUNICIPAL DE VITÓRIA DA CONQUISTA (PMVC). Secretaria da Transparência e do Controle. Carta de Serviços aos Usuários. Vitória da Conquista - BA, 2020. Disponível em: < https://www.pmvc.ba.gov.br/wpcontent/uploads/CARTA-FINAL-23.01-1.pdf >. Acessado em 25 de novembro de 2020.

15. SCHIMITH MD, et al. Acessibilidade organizacional: barreiras na continuidade do cuidado na Atenção Primária à Saúde. Revista de Enfermagem da UFSM, 2019; 9:17.

16. SAMPAIO MVDA. Acesso à atenção especializada na região de saúde de Vitória da Conquista/BA e a garantia do direito à integralidade na Atenção à Saúde. Dissertação (Mestrado) - Curso de Saúde Pública - Curso de Saúde Pública, Escola Nacional de Saúde Pública Sergio Arouca, Fiocruz - Fundação Oswaldo Cruz, Vitória da Conquista Bahia, 2016; 22.

17. SANTOS L. Região de saúde e suas redes de atenção: modelo organizativo-sistêmico do SUS. Ciência \& Saúde Coletiva, 2017; 22: 1281-1289.

18. SERRA CG, RODRIGUES PHDA. Avaliação da referência e contrarreferência no Programa Saúde da Família na Região Metropolitana do Rio de Janeiro. Ciênc. saúde coletiva, 2010; 15(3): 3579-3586.

19. XIMENES NETO FRG, et al. Reflexões sobre a formação em Enfermagem no Brasil a partir da regulamentação do Sistema Único de Saúde. Ciência \& Saúde Coletiva, 2019; 25: 37-46. 